\title{
LONG PULSED LASER INDUCED REVERSE BULGING AND PLUGGING
}

\author{
Y. C. ZHOU, ${ }^{\dagger}$ Z. P. DUAN and B. M. XIE \\ Laboratory for Laser and Dynamic Behaviors of Materials, Institute of Mechanics, CAS, Beijing, 100080 , \\ People's Republic of China
}

\begin{abstract}
A new kind of failure mode is observed in circular brass foils whose peripheries are fixed and whose surfaces are subjected to a long pulsed laser over a central region. The failure is classified into three stages; they are referred to as thermal bulging, localized shear deformation and perforation by plugging. A distinct feature of the failure mode is that bulging and plugging occurred in the direction opposite to the incident laser beam. The failure mode is different from the well-known types of laser induced material damage, such as spallation, melting and/or vaporization. (c)1997 Elsevier Science Ltd.
\end{abstract}

\section{INTRODUCTION}

Interaction of high power laser with materials leads to two types of coupled damage, namely mechanical and thermal damage, depending on laser parameters and target material mechanical properties [1-8]. As far as laser parameters are concerned, the intensity $I$ and duration $\tau$ are usually considered as two main governing factors, of which the different values could produce different types of damage [3,4]. A brief description of damages was given in [4]. When the laser intensity is so high and the duration so short that the plasma is formed on the surface of the material, a shock wave propogates into the material and the wave reflection takes place between two surfaces. There is a potential of failure by spallation due to the wave reflection [6-8]. When the laser intensity is moderate, the material can either be predominantly melted to form a crater in the material [9], or can be predominantly vaporized to cause evaporative damage [10]. When the laser intensity is low, thermal stress induced by non-uniform thermal expansion seems a unique source, which could produce a thermal elastic-plastic deformation in the heat affected zone [11].

However, in the previous studies on laser-induced material damage, researchers more-or-less ignored the fact that the spatial shapes of the laser beam could also play an important role in controlling the failure mode. Those situations prevail where the size and intensity of the laser beam are such that the spatial structure effect will contribute to the mode of failure such as bulging followed by plugging. Such are the cases considered in this investigation.

\section{EXPERIMENTAL PROCEDURE}

Consider an incident laser beam that impinges normally on a circular brass foil as shown in Fig. 1. The $z$ - and $r$-directions are aligned normal and parallel to the specimen whose edge is clamped. Let $2 a$ denote the diameter of the laser spot that can vary from $2 \mathrm{~mm}$ to $6 \mathrm{~mm}$. The foil has a diameter of $30 \mathrm{~mm}$ and thickness of $0.1 \mathrm{~mm}$. The brass material contains $65 \%$ copper, $33.6 \%$ zinc, $0.03 \%$ iron, $0.06 \%$ antimony and other microelements that are negligibly small in percentage by weight.

The energy of the laser beam ranges from $25 \mathrm{~J}$ to $40 \mathrm{~J}$ with an intensity of the order of $10^{5}$ to $10^{6} \mathrm{~W} / \mathrm{cm}^{2}$. It is a single pulse Nd:grass laser with a wavelength of $1.06 \mu \mathrm{m}$. The optical path and devices arrangement are shown schematically in Fig. 2. The diagnostics of the laser parameters

† Also at the Department of Physics, Xiangtan University, 411105 Xiangtan Hunan, People's Republic of China. 


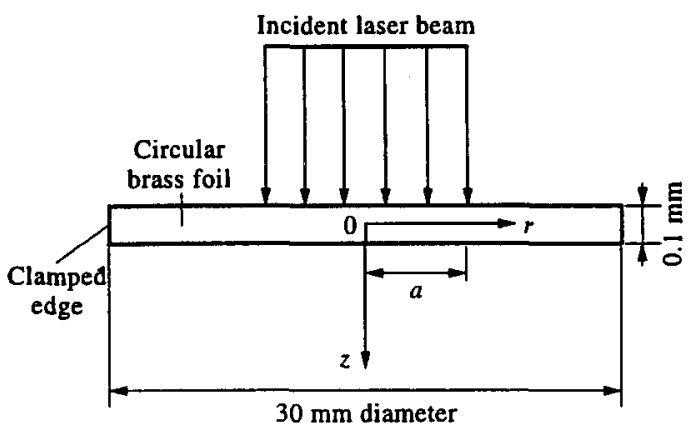

Fig. 1. Schematic of a normal incident laser beam imping on a circular brass foil specimen.

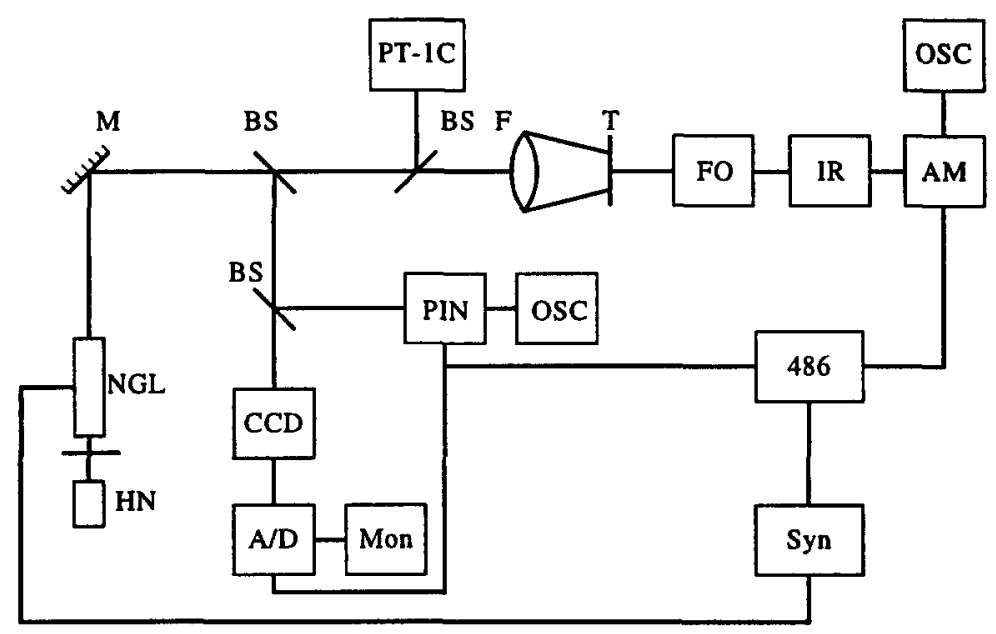

Fig. 2. Optical path and device arrangement in the test. NGL=Nd:grass laser; $H N=H e-$ $\mathrm{Ne}$ laser; $\mathrm{M}=$ mirror; $\mathrm{BS}=$ beam splitter; $\mathrm{CCD}=$ charge-coupled device sensor; $\mathrm{A} / \mathrm{D}=\mathrm{A} / \mathrm{D}$ transformer; Mon=monitor; PIN=PIN photoelectric cell; OSC=oscillograph; PT-1C=PT-1C laser energy recorder; $\mathrm{F}=$ focalizer $(f=100 \mathrm{~mm}) ; \mathrm{T}=$ target; $\mathbf{F O}=$ focusing system for infrared rays; $\mathrm{IR}=\mathrm{InSb}$ infrared detectors; $A M=$ amplifier; $486=486$ computer; $S y n=$ synchronizer.

provide a traditional monitoring of the laser beam characteristics, such as energy, temporal and spatial shapes. A photoelectric cell with the corresponding response time less than $1.0 \mu \mathrm{s}$ was used to measure the temporal shape of the laser beam. The spatial shape of the laser intensity was detected by charge-coupled devices (CCD) sensors. In experiment, the temperature rise on the rear surface was measured using a focused imaging system of infrared detectors, of which the focusing system was designed to be different from that used by Duffy et al. [12] and Zehnder and Rosakis [13]. The detailed information on the temperature measurement will be given elsewhere.

Displayed in Fig. 3 is the normalized intensity $I / I_{\max }$ with time. The normalized intensity $I / I_{\max }$ with space coordinates $r / a$ is shown in Fig. 4, where $r$ and $a$ are, respectively, the radial distance and the radius of the laser spot. The full width at half of the maximum laser is approximately $250 \mu \mathrm{s}$. The laser intensity rises rapidly within $50 \mu$ s and then decays exponentially with a sawtooth oscillation. The spatial distribution of the laser intensity is non-Gaussian but roughly uniform within the laser irradiated region and declines very sharply towards the edge where the laser spot terminates. For the convenience of numerical analysis, the laser intensity $I$ is approximated by

$$
I=I_{\max } \mathrm{e}^{-\alpha t}\left(1-\mathrm{e}^{-\beta t}\right) f(r)=I_{\max } g(t) f(r)
$$

where $\alpha=1.5 \times 10^{4} \mathrm{~s}^{-1}$ and $\beta=8 \times 10^{4} \mathrm{~s}^{-1}$ are determined experimentally. Therefore, the laser energy $E_{J}=\beta \pi a^{2} I_{\max } / \alpha(\alpha+\beta)$ and we have 


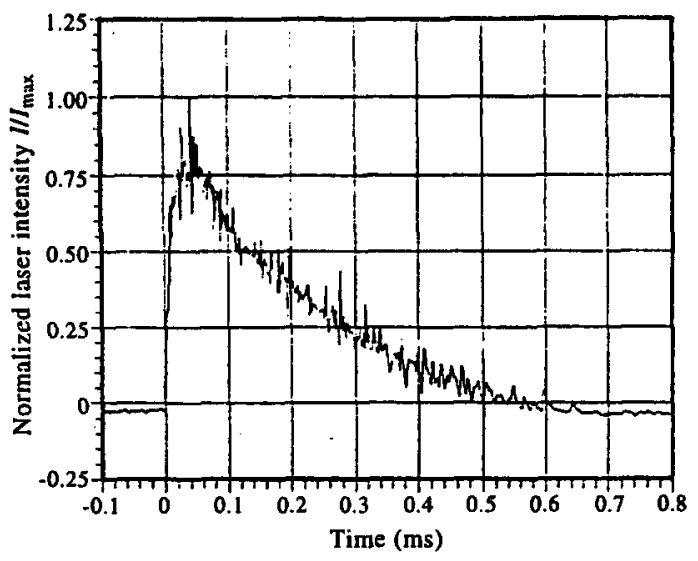

Fig. 3. Temporal shape of Nd:grass pulsed laser intensity.

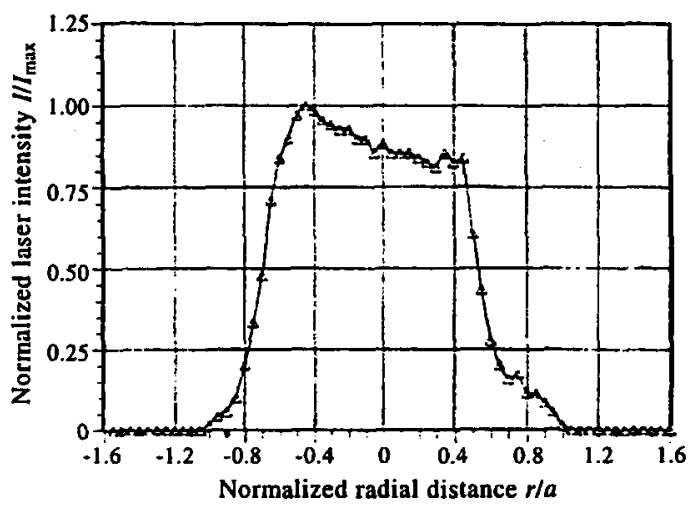

Fig. 4. Spatial shape of Nd:grass pulsed laser intensity.

$$
f(r)= \begin{cases}1 & 0 \leq r<a \\ 0 & a \leq r<\infty\end{cases}
$$

and $f(r)=\mathrm{e}^{-(r / a)^{2}}$ to account for the non-Gaussian and Gaussian nature of the laser beam, respectively.

\section{EXPERIMENTAL RESULTS}

\subsection{Description of failure mode}

Illustrated schematically in Fig.5 is the evolution of specimen failure. Figure 5(a) shows bulging of the brass foil at the earlier stage of laser irradiation. Note that this occurs towards the side of the incident laser beam where the temperature would be higher. Considerable shear deformation occurs around a rim near the edge of the laser beam which is shown in Fig. 5(b). This leads to the softening of the material due to intense heating.

Further intensification of the energy around the periphery of the laser beam leads to the initiation of plugging and final perforation which are shown in Fig. 5(c) and (d), respectively. This plugging mode of failure is customarily known to be associated with metal projectiles penetrating through metal targets in plate form [14,15]. A plug of the target material is ejected in the direction of the energy source that is the moving projectile. In the case of an incident laser beam, plugging occured in opposition to the incoming direction of the energy source. The initial bulge occurs on the side with the higher temperature, which determines the direction of plugging. 

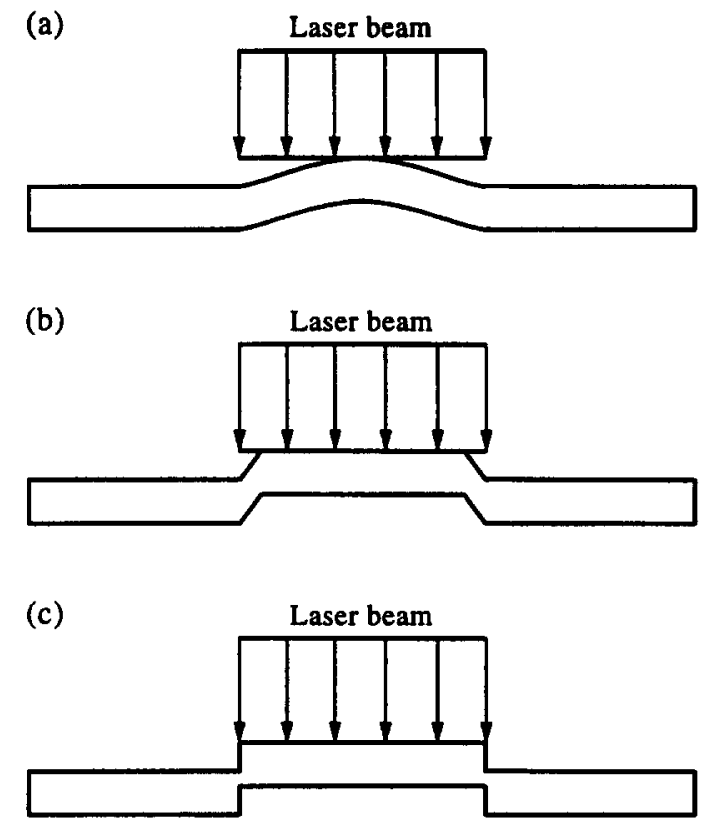

(d)

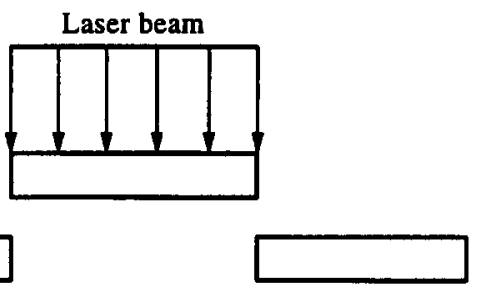

Fig. 5. Schematic of damage evolution: (a) bulging; (b) localized shear deformation; (c) plugging initiation; (d) perforation.

\subsection{Macro-phenomena and threshold intensity}

According to the test data given in Table 1 when the laser energy density is lower than $151 \mathrm{~J} / \mathrm{cm}^{2}$, or equivalently, the laser intensity is less than $0.51 \times 10^{6} \mathrm{~W} / \mathrm{cm}^{2}$, no visible macroscopic damage was observed on the front or rear surface of the brass specimen. When the laser intensity is increased to $0.56 \times 10^{6} \mathrm{~W} / \mathrm{cm}^{2}$, the bulging of the brass specimen is observed. A slight melting is observed on the front surface at the periphery of the laser spot. The laser intensity threshold value $I_{c r}$ for plugging to occur is about $0.61 \times 10^{6} \mathrm{~W} / \mathrm{cm}^{2}$. As $I$ exceeds $I_{c r}$, local melting of the material begins to take place. When the laser intensity is increased to $0.75 \times 10^{6} \mathrm{~W} / \mathrm{cm}^{2}$, the brass foil is totally fractured and fragmented. A study on laser-induced spallation in $0.1 \mathrm{~mm}$ thick copper foil has been made in [7]. The intensity was of the order of $5.3 \times 10^{10} \mathrm{~W} / \mathrm{cm}^{2}$; it is different by five orders of magnitude to failure by plugging, which is about $0.71 \times 10^{6} \mathrm{~W} / \mathrm{cm}^{2}$. The thresholds for the laser energy do not distinguish the two different failure modes as they are both approximately equal to $210 \mathrm{~J} / \mathrm{cm}^{2}$.

\subsection{SEM observations}

3.3.1. Bulging and localized shear deformation. The photograph of a polished section of brass specimen shown in Fig. 6 bulging in the direction opposite to the laser beam is observed with a maximum center deflection of $0.03 \mathrm{~mm}$. This corresponded to a laser energy of $8.2 \mathrm{~J}$; an intensity greater than $0.61 \times 10^{6} \mathrm{~W} / \mathrm{cm}^{2}$; and a spot diameter of $2.3 \mathrm{~mm}$. Softening of the material prevailed around the outer edge of the laser spot. 
Table 1. Macrophenomena and threshold intensity

\begin{tabular}{|c|c|c|c|c|c|c|}
\hline No. & $\begin{array}{c}\text { Energy } \\
E_{J} \\
(\mathrm{~J})\end{array}$ & $\begin{array}{l}\text { Laser spot } \\
\text { diameter } \\
(\mathrm{mm})\end{array}$ & $\begin{array}{l}\text { Energy } \\
\text { density } \\
\left(\mathrm{J} / \mathrm{cm}^{2}\right)\end{array}$ & $\begin{array}{c}\text { Intensity } \\
\left(\times 10^{6} \mathrm{~cm}^{-2}\right)\end{array}$ & $\begin{array}{c}\text { Spallation threshold } \\
\left(\mathrm{J} / \mathrm{cm}^{2}\right) \\
(\mathrm{T}=4.0 \mathrm{~ns})[7]\end{array}$ & Macrophenomena \\
\hline 1 & 29.6 & 5.0 & 151 & 0.51 & 210 & No observable macrodamage \\
\hline 2 & 33.2 & 5.0 & 169 & 0.56 & 210 & $\begin{array}{l}\text { At the periphery of the laser } \\
\text { spot, a slight melting is observed } \\
\text { on the front surfaces of the brass } \\
\text { foil. The laser irradiated region } \\
\text { bulges in the opposite direction } \\
\text { to the incident laser }\end{array}$ \\
\hline 3 & 35.7 & 5.0 & 182 & 0.61 & 210 & $\begin{array}{l}\text { A slight melting is observed } \\
\text { on the front surface at the } \\
\text { periphery of the laser spot. } \\
\text { Plugging takes place opposite to } \\
\text { the direction of the incident laser } \\
\text { beam }\end{array}$ \\
\hline 4 & 38.3 & 5.0 & 195 & 0.65 & 210 & As for (3) above \\
\hline 5 & 41.6 & 5.0 & 213 & 0.71 & 210 & $\begin{array}{l}\text { The laser irradiated part simply } \\
\text { separated from its edge }\end{array}$ \\
\hline 6 & 44.1 & 5.0 & 226 & 0.75 & 210 & Fragmented completely \\
\hline
\end{tabular}

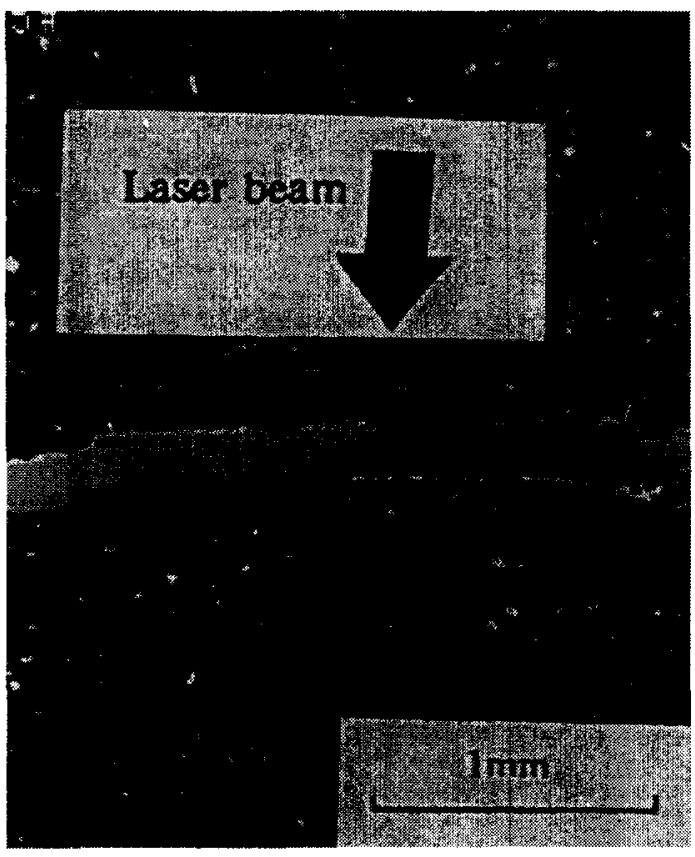

Fig. 6. Sectioned brass foil at the bulge state with a laser energy of $8.2 \mathrm{~J}$ over an area of $2.3 \mathrm{~mm}$ in diameter.

3.3.2. Cracks and fractographs. The fractographs and the microcracks by plugging are shown in Fig. 7. This corresponded to a laser energy of $29 \mathrm{~J}$ and a spot diameter of $4.5 \mathrm{~mm}$. Figure 7(a) shows the fractograph in the circular brass foil failed by plugging in opposition to the direction of the incident laser beam. Also the photograph of the enlargement of area $D$ in Fig. 7(a) is shown in Fig. 7(b). The microcracks and macrocrack in the peripheral region are marked with arrows $B$ and $C$ respectively in Fig. 7(c). Figure 8 shows the microcrack on the rear surface at the periphery of the laser spot. Figures 7 and 8 show that increased laser intensity leads to the initiation of microcracks in the peripheral region with radius $r \sim a$. The macrocracks appeared on the rear surface and then spread into the material. They would grow and coalesce into macrocracks that grow rapidly in the circumferential direction as well as in the thickness direction.

In addition, the fractographs induced by the laser beam plugging and by metal projectile pene- 

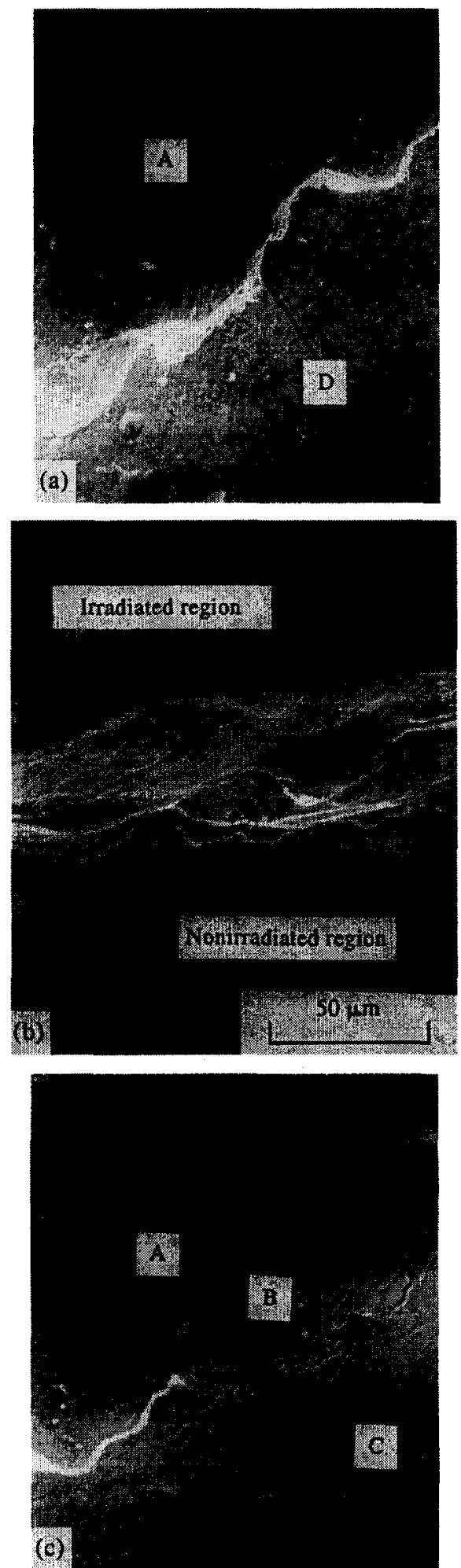

Fig. 7. Fractographs and cracks viewed from the rear surfaces: (a) fractographs caused by plugging; (b) enlargement of area $D$ on a photograph of (a); (c) microcracks marked with arrow $B$, and macrocracks marked with arrow $C$, appearing on the rear surface and then growing in the circumferential direction-the regions marked by $A$ in (a) and (c) denote the rear surface of brass foil and their front surfaces are subjected to a laser energy of $31 \mathrm{~J}$ over a region of diameter

$$
4.5 \mathrm{~mm} \text {. }
$$




\section{Laser beam}

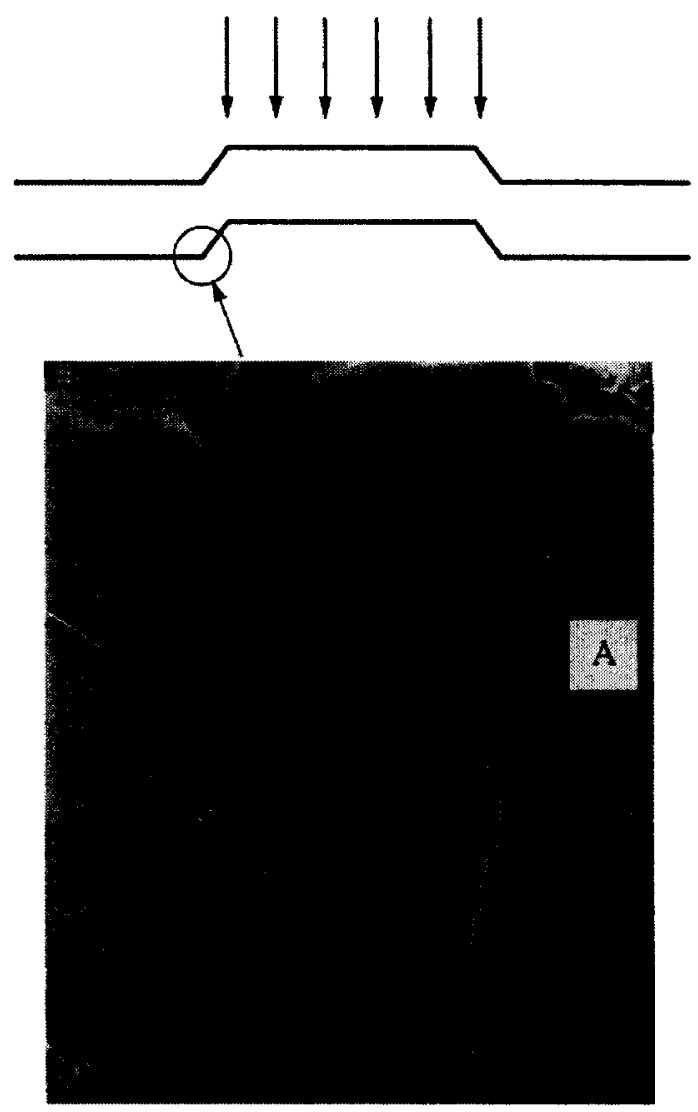

Fig. 8. Sectioned brass foil for the initiation of microcrack, as marked with arrow $A$, on the rear surface and then growing in the thickness direction.

trating are shown in Fig. 9(a) and (b), respectively. Figure 9(a) corresponds to a laser energy of $29 \mathrm{~J}$ and a spot diameter of $4.5 \mathrm{~mm}$. Figure 9 (b) corresponds to a brass plate of thickness $1.0 \mathrm{~mm}$ perforated by a metal projectile of length $10 \mathrm{~cm}$ which moved with velocity $4.79 \mathrm{~m} / \mathrm{s}$. The experiment was carried out using a split Hopkinson pressure bar. The characteristics for the fractographs do not distinguish the two different failure modes.

3.3.3. Fragmentation. When the laser intensity is increased to $0.75 \times 10^{6} \mathrm{~W} / \mathrm{cm}^{2}$, the brass foil is totally fractured and fragmented. Figure 10 shows a section of fracured and fragmented brass foil which has been subjected to a laser energy of $9.2 \mathrm{~J}$ over a region of diameter $2.3 \mathrm{~mm}$.

\section{DISCUSSIONS}

The observed plugging failure of the brass foil specimen subjected to the Nd:grass long pulsed laser can be summerized in three stages.

\section{Stage 1}

The brass foil bulges towards the laser beam. At the earlier stage of laser irradiation, the deformation of the brass foil is infinitesimal and the classical Kirchhoff plate theory can be used to determine the deflection $w(r, t)$. Then we have the equivalent transverse loading $q_{\theta}$ as [16] 

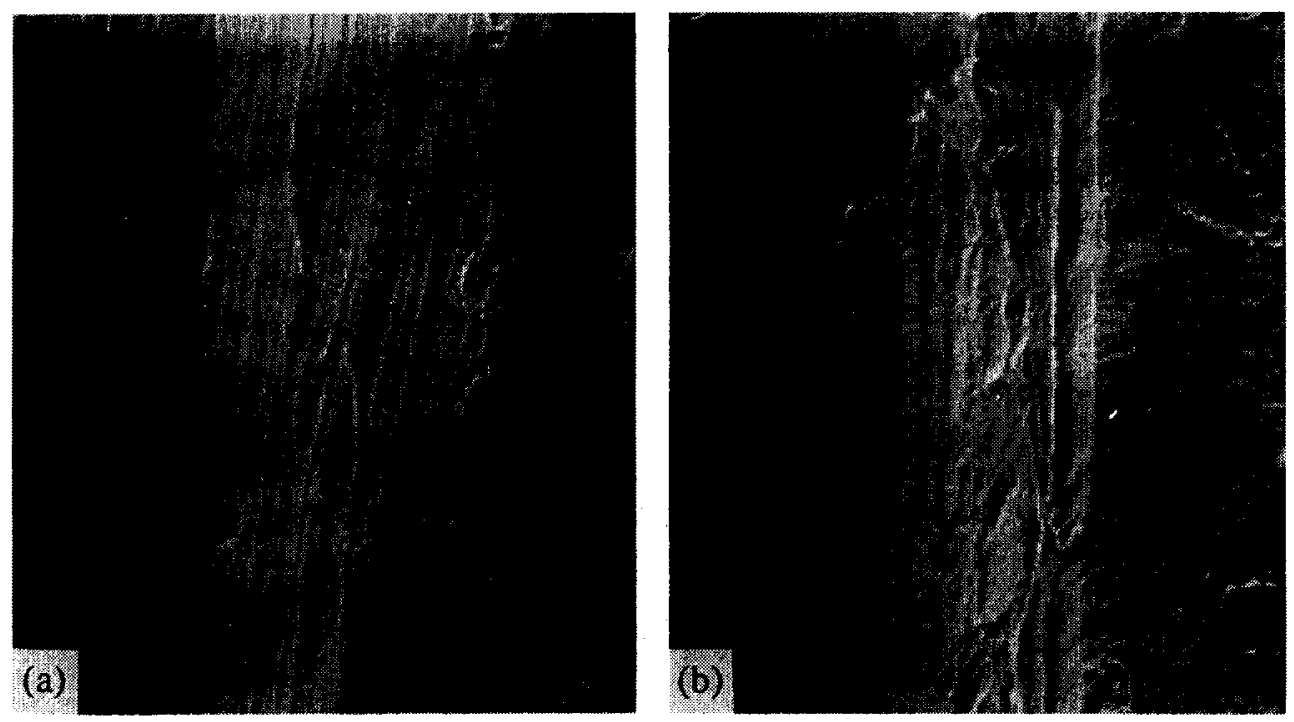

Fig. 9. Fractographs induced by plugging: (a) fractograph in brass foil subjected to a laser energy of $29 \mathrm{~J}$ over a region of diameter $4.5 \mathrm{~mm}$; (b) fractograph in brass plate of thickness $1.0 \mathrm{~mm}$ perforated by metal projectile of length $10 \mathrm{~cm}$ which moved with velocity $4.79 \mathrm{~m} / \mathrm{s}$.

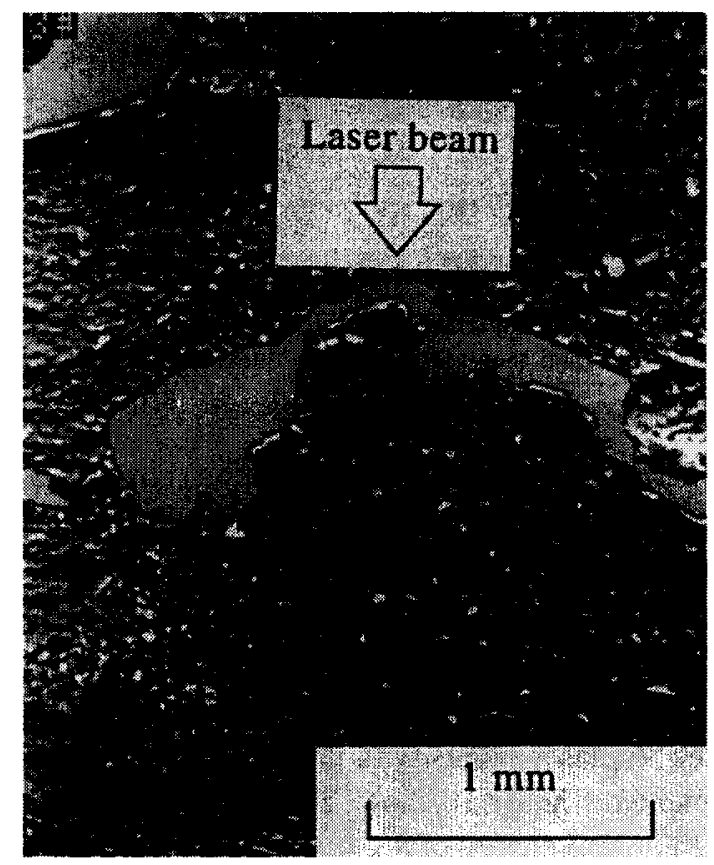

Fig. 10. Fractured and fragmented brass foil subjected to a laser energy of $2.9 \mathrm{~J}$ over region of diameter $2.3 \mathrm{~mm}$.

$$
q_{\theta}=-\frac{1}{1-v} \Delta^{2} M_{\mathrm{T}}
$$

where $M_{\mathrm{T}}$ is the thermal moment

$$
M_{\mathrm{T}}=\alpha_{0} E \int_{-h / 2}^{h / 2} \theta(r, z, t) z \mathrm{~d} z
$$

and $\nu, E, \alpha_{0}$ and $h$ are, respectively, Poisson's ration, Young's modulus, the thermal expansion coefficient and the brass foil thickness. In the above expressions, $\Delta^{2}=\frac{d^{2}}{d r^{2}}+\frac{1}{r} \frac{d}{d r}$ is the Laplace 
operator and $\theta(r, z, t)$ is the temperature rise.

A high temperature state is built up on the specimen front surface impinged by a normal incident laser beam. This means that the temperature gradient in the $z$-direction, i.e. $\frac{\partial \theta}{\partial z}$ or $M_{\mathrm{T}}$, is high at the earlier stage of laser irradiation and tends to zero at the later stage. The spatial profile of $M_{\mathrm{T}}$ is almost the same as that of the laser beam. The result of this is that $q_{\theta}$ is consistently negative in the irradiated region and consistently positive near the outer edge of the laser beam. However, in the case of metal projectiles penetrating through metal targets in plate form, the transverse loading is consistently positive and zero within and without the projectile's penetrating region, respectively. The negative value of $q_{\theta}$ in the irradiated region causes the foil to bulge towards the direction opposing the laser irradiation.

\section{Stage 2}

Higher shear deformation occurs around a rim near the outer edge of the laser beam. A steep temperature gradient prevails across the periphery of the laser beam where the spot terminates. The distinct feature of the spatial distribution of the laser intensity, or equivalently the distinct feature of the spatial distribution of the equivalent transverse loading $q_{\theta}$, causes the shear strain at the periphery of the laser spot which is much larger than that in other regions [11]. High shear deformation concentrated in a rim is developed and causes a circular region impinged upon by the laser to move further in the bulged direction.

\section{Stage 3}

When the laser intensity reaches a critical value, failure by plugging takes place in opposition to the direction of the incident beam.

On a microscopic scale, the plugging failure characteristics induced by a laser are similar to those found in brass plates perfected by metal projectiles. Such experiments are well-known and have been carried out using a split Hopkinson pressure bar. To reiterate, laser-induced failure by plugging depends on the incident laser intensity and duration, the spatial profile of the laser beam and their relation to the geometric and material characteristics of the specimen.

Acknowledgements-The authors would like to express their sincere thanks to Professor Z. M. Chen for his many helpful discussions during the preparation of this paper. Support for this research program was provided partly by NNSF of China and partly by the Field of Laser Technology, 863-NHT Research Development Program. Their support is also gratefully acknowledged.

\section{REFERENCES}

1. Ready, J. F., Effects of High-Power Laser Radiation. Academic Press, New York, 1971.

2. von Allmen, M., Laser-Beam Interactions with Materials-Physical Principles and Applications. Springer-Verlag, New York, 1987.

3. Wendlandt, B. C. H., Theoretical Studies of the Interation of High-Power Laser Beam and Metals. NASA-74-16188, 1973.

4. Kar, A. and Mazumder, J., J. Appl. Phys., 1990, 68(8), 3884.

5. Fox, J. A., Appl. Phys. Lett., 1973, 22(11), 594.

6. Hermann, J., Boulmer-Leborgne, C., Dubreuil, B. and Mihailescu, I. N., J. Appl. Phys, 1993, 74(5), 3071.

7. Eliezer, S., Gilath, I. and Bar-Noy, T., J. Appl. Phys., 1990, 67(2), 715.

8. Boustie, M. and Cottet, F., J. Appl. Phys., 1991, 69(11), 7533.

9. Zweig, A. D., J. Appl. Phys., 1991, 70(3), 1684.

10. Chan, C. L. and Mazumder, J., J. Appl. Phys., 1987, 62(11), 4579.

11. Medford, J. E. and Gray, P. M., The Response of Structural Materials to Combined Laser and Mechanical Loading. AIAA-80-1550, 1980.

12. Hartley, K. A., Duffy, J. and Hawley, R. H., J. Mech. Phys. Solids, 1987, 35(3), 283.

13. Zehnder, A. T. and Rosakis, A. J., J. Mech. Phys. Solids, 1991, 39(3), 385.

14. Anderson, C. E. and Bodner, S. R., Int. J. Impact Engng, 1988, 7(1), 9.

15. Shoukry, M., Nair, S. and Kalpakjian, S., Int. J. Engng Sci., 1991, 29(9), 1035.

16. Duan, Z. P., Zhou, Y. C. and Xie, B. M., in Proc. IUTAM Symp. on Impact Dynamics, ed. Z. M. Cheng and Q. M. Tan. Peking University Press, 1994, p. 176.

17. Zhou, Y. C. and Duan, Z. P., Shear deformation analysis of laser-induced reverse plugging effect. Int. J. Nonlinear Mech., 1997 (to appear). 